\title{
UN CURRÍCULO DE CIENCIAS EQUILIBRADO DESDE LA PERSPECTIVA DE GÉNERO
}

\author{
SAHUQUILLO BALBUENA, E., JIMÉNEZ ALEIXANDRE, M.P., DOMINGO \\ OUVRARD, F. Y ÁLVAREZ LIRES, M. \\ ICE Universidade de Santiago de Compostela.
}

\section{SUMMARY}

As a part of a larger study, a textbook analysis was undertaken with science texts, looking for balance or bias related to gender. The results show a bias toward the interests and previous experiences of boys, as well as stereotypes in language and images. Following the perspective of gender -inclusiveness, a proposal is presented about dimensions that should be taken into account in a science curriculum intended to be friendly for both girls and boys.

\section{INTRODUCCIÓN: ¿POR QUÉ HAY QUE CAMBIAR LAS CIENCIAS?}

Durante la década de los 80 , Ia línea de investigación conocida en sus inicios como «niñas y ciencias» y más adelante como "género y ciencias" ha puesto de manifiesto que la enseñanza de las ciencias no se dirige a un sujeto neutro, ni lo hace con unos contenidos y metodología equilibrados, sino que, partiendo de los intereses y experiencia previa de los alumnos varones, diseña unas estrategias que, en la práctica, van marginando a las mujeres de las opciones científicas y técnicas, tanto académicas como profesionales. Esta discriminación, que se inscribe en la sociatización asimétrica que tiene lugar desde el nacimiento, y en concreto en la escuela (Subirats y Brullet 1988), reviste, en el caso de tas ciencias experimentales, aspectos específicos que han sido tratados por ejemplo por Kahle (1985), Kelly (1987), Spear (1987) y Whyte (1986).

Cabe subrayar que esta discriminación se produce sin que el profesorado sea consciente de realizarla o sin que las personas autoras de materiales curriculares tengan la intención de disuadir a las alumnas de las opciones científicas. La existencia de dos niveles de formulación del sujeto "estudiante", uno inconsciente, en el que "estudiante» = «estudiante varón», y otro consciente o explícito, en el que se afirma que las y los "estudiantes» de ambos sexos son iguales y reciben igual tratamiento en clase, se pone de manifiesto, en las entrevistas al profesorado realizadas en el proyecto IDEA (Jiménez 1991). Por ejemplo, una persona, docente en Formación Profesional, al preguntarle la entrevistadora sobre los sesgos de los libros de texto, contestó que no se había fijado nunca en este aspecto. Al hacerle notar la entrevistadora la ausencia de mujeres en las ilustraciones, el tipo de problemas, etc., reaccionó comentando que sería deseable un cambio en el sentido de que aparecieran más mujeres en las ilustraciones, argumentando que así los «alumnos» se interesarían más, porque se conseguiría picar su amor propio. Evidentemente, este alumnos no es un genérico comprensivo, sino que se refiere sólo a los varones.

El cambio de denominación de la línea, que en la actualidad es "género y ciencias», subraya que la cuestión afecta no sólo a las alumnas, sino que es una manifestación de las transformaciones (Kelly 1987) que ha experimentado la formulación del propio problema en estudio, que hace diez años era: ¿Por qué tan pocas mujeres hacen Ciencia? , ¿Qué hay que cambiar en las niñas para que se interesen por las Ciencias?, mientras que ahora se plantea como: ¿Qué debe cambiar en la forma de hacer Ciencia? (Rosser 1986), ¿Qué funciona mal en la enseñanza de las ciencias? En particular la línea de estudio 
sobre género y ciencias ha suscitado algunas de las críticas más oportunas tanto a la forma de planificar el currículo, como de organizar las clases y, en general, a Ia forma de enseñar Ciencias (Head 1989), así como a los criterios y métodos de evaluación (Parker y Rennie 1992). El análisis y la solución de los problemas que estas críticas plantean debería llevar a un cambio en la enseñanza de las ciencias que vaya mucho más allá de un apoyo específico a las niñas.

En nuestra opinión, este cambio de orientación discurre en paralelo con otros experimentados en el pensamiento feminista, superando el «paradigma de la debilidad» (Català y García 1990). De igual forma que Lerner (1990) en Historia defiende una reconceptualización que sitúe a las mujeres en el lugar central que han ocupado como agentes de la Historia, criticando la posición de considerarlas como víctimas, y que Amorós (1985) en Filosofía propone una reconceptualización del sujeto del pensamiento, nuestra crítica a la enseñanza de las ciencias no se plantea desde la perspectiva de que tiene que «ayudar» a las estudiantes mujeres a suplir sus «carencias», sino que proponemos que se tengan en cuenta las diferencias en la experiencia previa que las y los estudiantes de ambos sexos aportan a las clases de ciencias, aprovechando el área de experiencia femenina para la instrucción, igual que se ha hecho hasta ahora con la masculina. Entendemos que el resultado será enriquecedor para los dos grupos (y no sólo para las niñas).

Conviene hacer notar gue en una supuesta sociedad igualitaria, las experiencias previas y los intereses de niñas y niños no tendrían por qué ser diferentes, por lo que hay que situar nuestra propuesta en el marco de Ia sociedad actual.

En este trabajo nos referiremos a la forma en que se concreta esta perspectiva en el currículo de Ciencias, abordando:

- Los sesgos en los materiales empleados en la actualidad.

- El modelo de currículo coherente con la perspectiva coeducativa.

\section{- Algunos ejemplos de concreciones de este modelo.}

Hay otras cuestiones, estrechamente relacionadas con éstas que no tratamos aquí, como los sesgos androcéntricos en la investigación científica (Jiménez 1992), la actuación con el alumnado o la formación del profesorado. En otras palabras, para lograr un cambio habrá que actuar sobre un conjunto de dimensiones interrelacionadas, de las que aquí abordamos solamente la curricular.

\section{ESTEREOTIPOS EN LOS TEXTOS DE CIENCIAS: EL ESTUDIO «IDEA»}

El proyecto IDEA, financiado por el CIDE, pretendía estudiar algunos factores relacionados con la segrega- ción de opciones en Formación Profesional, analizando respuestas de estudiantes, de docentes y libros de texto. El análisis del material empleado en la instrucción, junto con el estudio de las actitudes y comportamiento del profesorado, proporcionan información sobre cómo se enseñan las Ciencias. Sin duda el material más estandarizado y empleado de forma más general son los libros de texto, y en Ios países anglosajones ya hace tiempo que se vienen realizando estudios sobre textos de Ciencias, especialmente en lo referente a las ilustraciones (Powell y García 1985). En España un estudio de gran interés es el de Garreta y Careaga (1987) sobre textos de EGB, pero sólo aborda las áreas de Lenguaje y Sociales, y hasta ahora carecíamos de análisis en el área de Ciencias.

\subsection{Metodología}

La mayor parte de los estudios realizados en otros países se refieren fundamentalmente a las ilustraciones (proporción de mujeres, roles en que se representan...), y al lenguaje. Consideramos que en el área de Ciencias es necesario introcucir también una tercera dimensión, relativa a los contenidos, siendo éste uno de los aspectos más originales de nuestro estudio. Como instrumento elaboramos una plantilla de análisis (Anexo I) que cubre las siguientes dimensiones:

\section{Contenidos}

Se analizan en los apartados 1 (texto), 4 (cuerpo humano), 5 (problemas) y 6 (actividades prácticas). Aunque el 1 incluye al 4 , consideramos que el cuerpo humano requería un análisis aparte. Dentro de los contenidos se abordan a su vez estas dimensiones:

- La relevancia de lo tratado para ambos sexos o sólo para uno, es decir, si son cuestiones que responden a los intereses de alumnas y alumnos, o sólo de un sexo. Hemos utilizado como indicadores: $a$ ) el tratamiento de los temas de Ciencia y Tecnología en el contexto de las necesidades personales y sociales, o en uno abstracto, y b) el uso de ejemplos en relación con las experiencias previas y juegos de niñas y niños, o sólo de un sexo. Se aborda en $1.4,4.3,5$ y 6 . En el punto 2, al tratar del modelo curricular que proponemos, se discute con más detalle esta dimensión.

- La presentación de las y los profesionales de la Ciencia, utilizando como indicadores: $a$ ) citas de mujeres científicas, y $b$ ) tratamiento dado a Marie Sklodowska (Curie). Apartados 1.1 y 1.2

- El tratamiento dado a ambos sexos del ser humano, equilibrado o androcéntrico (varón como norma), tanto en general (1.3) como en el cuerpo humano (4.2 y 4.4)

\section{Lenguaje}

Aunque analizamos por separado el lenguaje empleado en el texto, en los problemas y en las prácticas, presentamos los resultados conjuntamente, aI no aparecer diferencias entre estos apartados. 
En cuanto al lenguaje, la dimensión más importante que se analiza es el empleo de términos genéricos, en la línea de las orientaciones ministeriales (MEC 1989), lo que hemos denominado lenguaje «coeducado», o la utilización del masculino como genérico (2.1) Además hemos analizado la presencia o ausencia de estereotipos asociados a actividades, profesiones, etc., y los saltos semánticos (2.2).

\section{Ilustraciones}

Las dimensiones abordadas son:

- La proporción de mujeres y niñas en dibujos y fotografías (3.1); el tema del cuerpo humano se ha analizado aparte (4.1).

- Los roles en que se representa a mujeres y niñas comparados con los roles en que se representa a los varones (3.2).

- La relevancia de las ilustraciones de experiencias, aparatos, situaciones, y en general las ilustraciones en que no hay personas, para ambos sexos.

En conjunto entendemos que con estas tres dimensiones se cubre de forma bastante completa el análisis de materiales.

\subsection{Muestra}

La muestra analizada ha sido seleccionada teniendo en cuenta los siguientes criterios:

- que cubriese todo el rango de edades, desde el momento en que las Ciencias aparecen como área separada, en los últimos cursos de EGB, hasta los últimos de Secundaria;

- que en Secundaria comprendiese textos tanto de BUP como de FP, y tanto de Física y Química como de Ciencias Naturales;

- que incluyese los textos más empleados en la actualidad, además de los utilizados en los centros de la muestra del estudio IDEA (centros de EGB y FP).

En total la muestra comprende 16 textos: 6 de Ciencias de $7^{\circ}$ y $8^{\circ}$ de EGB, 4 de diferentes asignaturas (Física y varias Tecnologías) de FP l, y 6 de diferentes asignaturas de BUP (Ciencias Naturales de $1^{\circ}$ y $3^{\circ}$ y Física y Química de $2^{2}$ ). En la memoria del estudio IDEA aparece una relación completa de los mismos y un análisis más detallado de los resultados.

\subsection{Resultados}

Al comentar los resultados hay que tener en cuenta que no todas las dimensiones estudiadas son aplicables a los

- 16 textos; por ejemplo, el cuerpo humano sólo aparece tratado en 4.

\section{Contenidos}

En la tabla I figuran los resultados en cuanto a los contenidos. Discutiremos cada una de las dimensiones por separado:

- En cuanto a la relevancia de los temas y ejemplos, en todos los textos predominan los que interesan más a los varones. El tratamiento es impersonal, y los conceptos científicos se presentan descontextualizados, sin relación con la vida cotidiana. Por ejemplo, en los temas de electricidad no se hace referencia a aparatos de uso doméstico; al tratar de la presión se habla de manómetros y columnas de mercurio, no de una olla a presión; no se da importancia a las aplicaciones de minerales y rocas, sino a su composición; etc.

Tabia I

Resultado del análisis de contenidos en textos de Ciencias.

\begin{tabular}{|c|c|c|c|c|c|}
\hline Dimenston & $\begin{array}{l}\text { Ndetextos } \\
\text { aplicable }\end{array}$ & & & & \\
\hline $\begin{array}{l}1.4 \\
\text { RELEVANCIA } \\
\text { CONTENIDOS }\end{array}$ & 16 & \multicolumn{2}{|c|}{$\begin{array}{c}\text { para nithas y ninos } \\
0\end{array}$} & \multicolumn{2}{|c|}{$\begin{array}{c}\text { mas para ninos } \\
16\end{array}$} \\
\hline $\begin{array}{l}5 \text { RELLVVANCIA } \\
\text { PROBLEMAS }\end{array}$ & 6 & \multicolumn{2}{|c|}{$\begin{array}{c}\text { para mitras y nlfios } \\
30 \\
\text { media } 5\end{array}$} & \multicolumn{2}{|c|}{$\begin{array}{c}\text { despersonalizados } \\
123 \\
\text { media } 20.5\end{array}$} \\
\hline $\begin{array}{l}6 \text { RELEVANCIA } \\
\text { PRACTICAS }\end{array}$ & 14 & \multicolumn{2}{|c|}{$\begin{array}{c}\text { para nifas } y \text { niftos } \\
75 \\
\text { media } 5,3 \\
\end{array}$} & \multicolumn{2}{|c|}{$\begin{array}{c}\text { despersonalizados } \\
187 \\
\text { redia 13,3 } \\
\end{array}$} \\
\hline $\begin{array}{l}1.1 \\
\text { PROFESIONALES } \\
\text { CENCIA }\end{array}$ & 16 & $\begin{array}{c}\text { total citas } \\
400\end{array}$ & $\begin{array}{c}\text { mujeres } \\
5\end{array}$ & & $\begin{array}{r}\text { varones } \\
395\end{array}$ \\
\hline $\begin{array}{l}1.3 \\
\text { TRATAMIENTO } \\
\text { AMBOS SEXOS } \\
\end{array}$ & 12 & $\begin{array}{c}\text { equilibrado } \\
0\end{array}$ & $\begin{array}{c}\text { mujer }=\text { nor } \\
0\end{array}$ & ma & $\begin{array}{c}\text { varón }=\text { norma } \\
12\end{array}$ \\
\hline $\begin{array}{l}\text { 4.2 ORDEN AP. } \\
\text { REPRODUCTOR }\end{array}$ & 4 & $\begin{array}{c}\text { alternando } \\
0 \\
\end{array}$ & $\begin{array}{l}\text { femenino } \\
\text { antes } \\
0 \\
\end{array}$ & & $\begin{array}{l}\text { masculino } \\
\text { ances } \\
\end{array}$ \\
\hline $\begin{array}{l}\text { 4.3 CONTENIDOS } \\
\text { REPRODUCCIÓN } \\
\text { - Hormonas } \\
\text { - Sexualidad } \\
\text { " Anticonceptivos } \\
\text { y ETS }\end{array}$ & 4 & $\begin{array}{c}\text { ambos sexas } \\
0 \\
0 \\
1\end{array}$ & $\begin{array}{c}\text { solo masctal } \\
1 \\
1 \\
0\end{array}$ & $2 n 0$ & \begin{tabular}{|} 
no lo trata \\
$\qquad \begin{array}{r}3 \\
3 \\
3\end{array}$
\end{tabular} \\
\hline
\end{tabular}

En los problemas y actividades sucede algo similar, y de los 25,5 problemas que hay, como media, por texto, sólo 5 pueden considerarse relevantes para ambos sexos, (hemos categorizado así aquéllos que proponen problemas o experiencias situadas en un contexto aplicado o de necesidades humanas, por ejemplo, un problema de cargas en la construcción de un edificio, y no sólo aquéllos - casi inexistentes- que se relacionan con actividades que se suponen «femeninas», como cocinar).

En las actividades prácticas, la proporción de las relevantes para ambos sexos es algo mayor, 5,3 de 18,6 como media por tex to, aunque el número de las despersonalizadas sigue siendo superior $(13,3)$.

- La presentación de las personas dedicadas profesionalmente a la ciencia es casi exclusivamente de varones: 
395 (a las que hay que af̂adir 95 en anexos), frente a sólo 5 de mujeres. Hay que señalar que, poniéndonos en la situación más desfavorable para nuestra hipótesis (que habría pocas citas de científicas), hemos contabilizado dos citas al «baño María», técnica alquimista inventada por María la Judía (Ogilvie 1986), aun cuando en los dos textos no se la menciona. Otra de las citas es de Meitner que aparece como el químico Meitner (cuando se desconoce el sexo de un científico se supone que es varón), y las dos restantes de Marie Curie, que esperábamos encontrar citada en cași todos los textos.

- El tratamiento dado a ambos sexos resulta, en todos los textos en que es aplicable (12), androcéntrico, considerando al varón como norma, puesto en evidencia en frases como la masa de un hombre medio es de $75 \mathrm{~kg}$ (que sólo sería adecuada si a lo largo del texto «hombre» fuese sinónimo de «varón» y no, como es el caso, de «ser humano»). Este androcentrismo es particularmente acusado en los temas del cuerpo humano, así también en ellas ocurre un importante cambio, después de describir los cambios experimentados por los varones en la pubertad; - Io mismo sucede en el gameto femenino, tras describir la gametogénesis en el caso masculino. En los 4 textos en que se trata el aparato reproductor, el masculino aparece en primer lugar, y en 2 con un texto más largo. EI único texto que menciona las hormonas sexuales, habla sólo de las masculinas, y lo mismo ocurre con la sexualidad. En un texto de EGB se ilustra la reproducción sexual con una lectura sobre la Mantis (la hembra mata al macho tras la cópula).

Es decir, en cuanto al contenido, los resultados confirman nuestra hipótesis de que se of rece una visión sesgada de la actividad científica como realizada por varones, con objetivos de carácter abstracto, sin relación con Ia sociedad humana, y considerando como sujeto de estudio, en lo que a la especie humana se refiere, fundamentalmente al varón.

Tabla II

Resultados del análisis de lenguaje en textos de Ciencias.

\begin{tabular}{|l|c|c|c|}
\hline \multicolumn{1}{|c|}{ Dimensión } & $\begin{array}{c}\text { N de textos } \\
\text { apifcable }\end{array}$ & equizibrada & estereotipada \\
\hline 2.1 & 16 & 0 & 16 \\
$\begin{array}{l}\text { *REFERENCIA A } \\
\text { PROFESIONES }\end{array}$ & 13 & 2 & 11 \\
$\begin{array}{l}\text {-REFERENCIA A } \\
\text { PERSONAS }\end{array}$ & & ausencla & estereotipos \\
\hline $\begin{array}{l}2.2 \\
\text { ESTEREOTYOS } \\
\text { SEXISTAS }\end{array}$ & 16 & 6 & 10 \\
\hline
\end{tabular}

\section{Lenguaje}

En la tabla II aparecen los resultados, en los que se han distinguido tres aspectos que corresponden en nuestra opinión a un sesgo más o menos acentuado del sexismo en el lenguaje.
Comenzando por el sesgo menos acentuado -o más generalizado-, el empleo del masculino como genérico en las profesiones y actividades: científicos, alumnos, observadores, geólogos etc., aparece en los 16 textos analizados. Decimos que éste es el sesgo «menor» porque su eliminación requiere, bien una perifrasis («profesionales de la investigación científica»), o ambos géneros («científicos y científicas»).

En las referencias a personas, hay 2 de los 13 textos a los que es aplicable que utilizan ser humano o persona, mientras que los demás hablan sistemáticamente de hombre, llegando a formulaciones imposibles como el hombre es viviparo.

El tercer aspecto, los estereotipos sexistas, es una categoría que hemos empleado cuando el sesgo va más allá del simple uso del masculino como genérico, y que aparece en 10 de los 16 textos. Algunos ejemplos son:

- Discriminar actividades o profesiones, por ejemplo médicos y enfermeras y no médicos y enfermeros, siguiendo la pauta empleada para las restantes profesiones.

- Emplear el genérico sólo para «ocuitar» el género femenino y no para incluir ambos géneros; así, en una misma página las personas utilizan, en un pie de ilustración que representa a una mujer, y los químicos investigan, en un pie de ilustración que representa a un varón. En ocasiones este empleo raya en la incoherencia, así, el primer niño probeta, en referencia a Louise Brown, que es niña.

- Connotaciones negativas en las únicas referencias femeninas en libros que emplean el masculino como genérico; así; mujeres dementes, y señoras encopetadas, en un libro que emplea constantemente el masculino.

En nuestra opinión, el conjunto de estos aspectos muestra sesgos en el lenguaje, algunos paralelos a los que aparecen en otras áreas y otros particulares del área de Ciencias.

Tabla III

Resultados del análisis de ilustraciones en textos de Ciencias.

\begin{tabular}{|l|c|c|c|}
\hline \multicolumn{1}{|c|}{ Dumensión } & $\begin{array}{c}\text { N de textos } \\
\text { aplicable }\end{array}$ & & \\
\hline $\begin{array}{l}3.1 \text { proporción } \\
\text { sexos } \\
4.1 \text { cuerpo } \\
\text { humano }\end{array}$ & 16 & equilibrada & más varones \\
\hline $\begin{array}{l}3.2 \text { roles } \\
\text { 3.3 relevanc1 } \\
\text { ejemplos }\end{array}$ & 8 & 1 & 16 \\
\hline
\end{tabular}

\section{Ilustraciones}

En la tabla III aparecen los resultados en cuanto a los tres aspectos que hemos mencionado más arriba. 
En cuanto a la proporción de ambos sexos, en los 16 textos hay más varones que mujeres, con proporciones que varían de 2 mujeres de 29 personas (texto con pocas figuras humanas), a 27 mujeres de 160 personas (texto con bastantes). También son masculinas las manos que aparecen realizando actividades, excepto unas femeninas que hacen jabón.

En particular, en los órganos y aparatos del cuerpo humano, sólo hay un texto (de 8), que podemos considerar equilibrado, mientras que en los demás las siluetas, Ios dibujos de músculos, etc., son masculinos, apareciendo ambos sexos sólo en la reproducción.

En cuanto a los roles de las personas, aplicable a 10 textos, sólo puede considerarse equilibrado uno, mientras que en los demás los varones aparecen realizando actividades variadas y las mujeres son representadas sólo como madres con bebés, enfermeras, amas de casa empleando electrodomésticos, etc. En varias escenas de trabajo en laboratorios, las mujeres miran a varones que trabajan. En algunos textos, las únicas mujeres que hacen algo distinto a tareas domésticas son de carácter exótico (una domadora hindú).

En cuanto a la relevancia de lo representado, igual queen la dimensión de contenido, los ejemplos son descontextualizados en 11 de los 12 textos. Así un termostato representado aislado (y no incluido en un radiador u otro aparato de uso diario).

\section{UN MODELO DE CURRÍCULO EQUI- LIBRADO}

El modelo de currículo de Ciencias que proponemos pretende ser equilibrado, es decir, interesar a niñas y niños, contar con la experiencia previa de ambos sexos, y promover expectativas similares para unas y otros, de forma que las adolescentes que tengan en perspectiva una opción profesional técnica o científica no se vean marginadas de ella, y que la mayoría del alumnado aprecie la relevancia de la ciencia y la técnica para su vida cotidiana y mantenga ante éstas una actitud racional y crítica, tan distante de la admiración reverencial hacia lo «científico» entendido como dogma indiscutible como del rechazo y el miedo hacia una actividad que no se comprende y que parece perjudicar a la humanidad.

Cambiar el modelo de currículo no es sólo tarea de las personas que escriben textos, o que los editan, aunque nuestro análisis se ha centrado en ellos. Puesto que el diseño curricular depende de la administración, también le cabe a ésta la responsabilidad en la selección de los temas y en las orientaciones sobre su tratamiento. $Y$, por stupuesto, sin una sensibilización del profesorado, que es quien pone en práctica los materiales en el aula, de poco servirían diseños curriculares y textos más equilibrados.

Entendemos que a partir de este momento podemos superar la etapa de crítica a lo existente, y proponer alternativas en las que se concrete el nuevo modelo. Por supuesto que la crítica es necesaria, porque la mayoría del profesorado no es consciente de que, por ejemplo, en los textos de Ciencias se produzca un ocultamiento de la actividad de las mujeres y de su propio cuerpo. Pero creemos que el trabajo realizado hasta ahora permite avanzar un paso más, y que este avance, en línea con propuestas como las de Harding (1986), Head (1989) y Smail (1991, esta última traducida al castellano sin el nombre de la autora), puede concretarse en el caso del currículo en aspectos como los que se sugieren más adelante. Los resultados de programas de intervención en esta línea (Head y Ramsder 1990) indican que las chicas mantienen o aumentan su interés por las Ciencias, y además que la asociación entre la elección de asignaturas de Ciencias y un determinado tipo psicológico desaparece; en otras palabras, estos programas hacen las Ciencias más atractivas para una mayor variedad de alumnas.

\subsection{Selección equilibrada de bloques y temas}

Esto significa que deben incluirse cuestiones que interesen a ambos sexos. Por ejemplo, los resultados del estudio GIST (Kelly 1988) en el Reino Unido, así como los de un trabajo realizado por nosotras (Álvarez y Jiménez 1992, Soneira 1992) muestran que los adolescentes varones se interesan más por el funcionamiento de máquinas y aparatos, y las adolescentes mujeres más por cuestiones relacionadas con la salud, el cuerpo humano, la influencia de la tecnología en la sociedad, etc. Hay que señalar que también a los chicos les interesan las cuestiones del cuerpo bumano.

Nuestra propuesta sería buscar los temas en que coincide un gran interés de ambos sexos, como «estrellas y planetas», «los alimentos más adecuados», «educación sexual» u «ordenadores».

\subsection{Contextualizar los temas en las necesidades personales y sociales}

Según muestran distintos estudios (Head 1989, Head y Ramsden 1990), parece que las mujeres se implican en mayor grado en los problemas científicos cuando éstos se presentan en el contexto de las necesidades de las personas y de la sociedad. Es đecir, que también lograremos interesar a las niñas si los temas como funcionamiento de máquinas, presión, electricidad, etc. se trabajan en relación con su uso cotidiano o con la utilidad que han tenido para la humanidad. Es preferible, en este sentido, que los ejemplos pongan de manifiesto la interaccion entre Ciencia, Tecnología y vida diaria, y que conecten con la experiencia previa de las alumnas y los juegos con los que están más familiarizadas.

\subsection{Incorporación de las aportaciones de las mujeres a la Ciencia}

Un aspecto de esta incorporación es, por supuesto, que aparezcan científicas en los textos; así la nueva versión del proyecto Nuffield (Lyth 1986) comienza con una unidad sobre el trabajo de tres científicas. Pero además 
se trata de incorporar las aportaciones de las mujeres a la Ciencia y la Técnica: el desarrollo de la agricultura, el empleo de plantas medicinales, la fabricación de jabón, etc. En los casos en que se introducen los métodos de trabajo de la Ciencia puede hacerse alguna referencia a las dificultades encontradas por las mujeres para practicarla (por ejemplo su exclusión de los estudios universitarios de Medicina, cuando había sido una actividad. practicada tradicionalmente por mujeres).

\subsection{Tratamiento equilibrado de ambos sexos}

En los aspectos en que hay diferencias anatómicas, físiológicas... habría que tratarlos de forma conjunta y equivalente, evitando presentar a las mujeres to a «mujeres y niños") como un caso particular, una desviación de la norma que sería el varón. Por ejemplo, en las tablas de pesos y medidas, en las de calorías relacionadas con la đieta; al mencionar el número de eritrocitos, de pulsaciones, etc.

\subsection{Lenguaje no sexista}

Evitar los estereotipos, por muy frecuentes que sean en el lenguaje, y las formulaciones despectivas hacia las mujeres. Tratar de no incurrir en saltos semánticos; incluir ambos sexos y otros aspectos en los que no entramos con detalle por ser comunes a todas las áreas.

\subsection{Equilibrio en las ilustraciones}

Incluir un número similar de mujeres y varones, niñas y niños, y representarlos realizando todo tipo de actividades, profesionales y técnicas (también las mujeres), o domésticás (también los varones), evitando los estereotipos como, por ejemplo, médico-enfermera, que ni siquiera corresponden a la realidad social española en la actualidad.

En las ilustraciones «no humanas», presentar ejemplos que conecten con la experiencia de ambos sexos, no sólo motos, automóviles o balones de fútbol, y aparatos e instrumentos técnicos contextualizados en situaciones lo más próximas posible a la vida diaria.

Creemos que estas propuestas pueden ser llevadas a la práctica en la actualidad, que son realizables y que no implican ninguna exageración, sino sencillamente corregir un enfoque androcéntrico sesgado que eliminaba a ias mujeres y todo lo relacionado con ellas de las actividades científicas.

\section{UNAS CIENCIAS MÁS ATRACTIVAS Y MÁS HUMANAS}

En el apartado anterior nos referíamos a la superación de una etapa en la que la mayor parte de los esfuerzos se dedicaron a criticar una forma de hacer Ciencia, de analizar la Historia de la Ciencia y de enseñar Ciencias impregnada de androcentrismo. De hecho un aspecto importante de esa crítica lo constituye el poner de manifiesto la existencia de estos sesgos, ya que para un gran número de personas esta situación era la «normal», y la propuesta de incorporar la perspectiva de las mujeres una pretensión poco razonable.

Una anécdota que revela hasta qué punto la invisibilidad de las mujeres es percibida como lo natural nos ocurrió con Ios cuestionarios de EGB del proyecto IDEA, en los que aparecían tres viñetas, dibujadas por Fran Jaraba, como introducción a las cuestiones. Varias personas, tanto profesorado de los centros de la muestra, como especialistas en educación nos comentaron que los dibujos eran chocantes porque había demasiadas mujeres. AI repetirse el comentario se nos ocurrió contar los personajes de las viñetas, con este resultado:

$\begin{array}{cccc}\text { Mujeres } & \text { Varones } & \text { Total } & + \text { (figuras pequeñas en el fondo) } \\ 5 & 9 & 14 & +(2 \text { varones })\end{array}$

Es decir, sin contar las figuritas masculinas del fondo, el número de varones es casi el doble que el de mujeres. Otra cuestión es el papel protagonista de las mujeres en esos dibujos, subrayado por el primer plano, y sin duda a eso se referían quienes nos decían que había algo raro.

Nuestro objetivo es llegar a una situación en la que no sea raro que aparezcan mujeres en Ios textos de Ciencias, porque haya mujeres ingenieras, electricistas, técnicas, mecánicas, etc. Por supuesto que esta propuesta pretende favorecer a las niñas, transformar la segregación de opciones que existe actualmente en las ramas de Formación Profesional y en las carreras de Ingeniería, y lograr que las niñas se interesen por las Ciencias y sigan escogiendo asignaturas y opciones relacionadas con ellas.

Pero además, los resultados del proyecto GIST (Whyte 1986) muestran que las modificaciones introducidas, tanto en el currículo como en la metodología de trabajo en clase, lograron un cambio de actitud también en los varones, e incluso en mayor grado en los adolescentes varones que en las adolescentes mujeres de los grupos experimentales. En otras palabras, estas Ciencias son más atractivas, y no sólo para las niñas, sino también para algunos niñ̃os que antes no conectaban con la forma tradicional de enfocar las clases. Como señalan Parker y Rennie (1992), la perspectiva de unas Ciencias equilibradas respecto al género comparte muchos aspectos con la perspectiva constructivista, e, indican estas autoras, el que estas perspectivas se lleven realmente a la práctica del aula depende en gran medida de que cambie también la forma de evaluar el aprendizaje de las Ciencias.

Desde que finalizó el GIST, ha habido otros proyectos de enseñar Ciencias sin sesgos de género, como el estudio realizado en el IPN (Hoffman et al. 1984), los trabajos del equipo australiano McClintock, Ia incorporación de esta dimensión en los materiales «Better Science» deI SSCR, o la nueva versión del Nuffield antes citada. Por nuestra parte, en el proyecto ACES (Otero et aI. 1991) 
estamos trabajando en el diseño de materiales para la Enseñanza Secundaria, en una perspectiva que contempla la Ciencia y la Tecnología en relación con las necesidades humanas. Aunque somos conscientes de que no es fácil romper con unos sesgos profundamente arraigados, creemos que es posible y que tendrá unos resultados más gratificantes tanto para las personas que aprenden como para las que enseñamos.

\section{REFERENCIAS BIBLIOGRÁFICAS}

ÁLVAREZ LIRES, M. y JIMÉNEZ ALEIXANDRE, M.P., 1992. Género, Ciencia y Tecnologia, en Moreno (ed.), Del Silencio a la Palabra. Coeducación y Reforma Educativa (Instituto de la Mujer: Madrid), pp. 178-196.

AMOROS, C., 1985. Hacia una crítica de la razón patriarcal. (Anthropos: Barcelona).

CATALÀ, A. y GARCÍA, E., 1990. ¿Qué quieres hacer de mayor? (Generalitat Valenciana; Institut Valencià de la Dona, Valencia).

GARRETA, N. y CAREAGA, P., 1987. El sexismo en el material escolar: los libros de texto actuales, en Primeras Jornadas Mujer y Educación. (Instituto de la Mujer, serie Documentos, n. 3: Madrid).

HARDING, J., 1986. Perspectives on Gender and Science. (The Falmer Press: Londres).

HEAD, J., 1989. The affective constraints on Learning Science, en Adey et al. (eds.), Adolescent Development and School Science. (The Falmer Press: Londres).

HEAD, J. y RAMSDEN, J., 1990. Gender, psychological type and science, Int.J.Sci. Educ, Vol. 12 (1), pp. 115-121.

HOFFMAN, L., LEHRKE, M. y TODT, E., 1984. Development and Change in Pupils' Interests in Physics (Grades 5 to 10) Design of a longitudinal study. 12th Symp. «Interests in Science and Technology Education». (IPN: Kiel).

JIMENEZ ALEIXANDRE, M.P., (coord.) 1991. Elección de ramas por las alumnas de Formación Profesional factores escolares relevantes en la actual segregación y líneas de modificación. Investigación financiada por el CIDE (Memoria final).

JIMÉNEZ ALEIXANDRE, M.P., 1992. Mujeres y Ciencia: superando el androcentrismo "Txostenak», Seminario de Estudios de la Mujer, Universidad. del País Vasco (en prensa).

KAHLE, J.B., 1985. Women in Science: a report from the Field. (The Falmer Press: London).

KELLY, A., 1987. Science for Girls? (Open University Press: Londres).

\section{AGRADECIMIENTOS}

EI proyecto IDEA fue llevado a cabo con financiación del Centro de Información y Documentación Educativa (CIDE) dentro del concurso nacional de Proyectos de Investigación Educativa 1989. Agradecemos también la concesión de una Acción Integrada Hispano-Británica, HB-023 en la convocatoria de 1990 que nos permitio trabajar con John Head sobre el modelo de currículo de Ciencias equilibrado.

KELLY,A., 1988. Getting the GIST: a Quantitative study of the Effects of the Girls into Science and Technology Project. (Dept. of Sociology Univ. of Manchester). Occasional Paper n. 22 .

LERNER, G., 1990. La Creación del Patriarcado. (Crítica: Barcelona).

LYTH, M., (ed). 1986. Nuffield Science 1) to 13. (Longman: Londres).

MEC, 1989. Recomendaciones para el uso no sexista de la lengua. (Madrid).

OGILVIE, M.B., 1986. Women in Science: Antiquity through the Nineteenth Century. (The MIT Press, Cambridge, Mass).

OTERO, L., JIMÉNEZ, M.P. y LORENZO, F.M., 1991. El proyecto ACES de Ciencia y Tecnología, Cuadernos de Pedagogía, n. 194, pp. 67-69.

PARKER, L. y RENNIE, L., 1992. Constructivism and GenderInclusiveness: Has assessment kept pace? Paper presented at the NARST meeting. (Cambridge, Mass).

POWELL, R. y GARCIA, J., 1985. The portrayal of minorities and women in selected elementary Science series, Journal of Research in Science Teaching, Vol. 22 (6) pp. 519-533.

ROSSER, S., 1986. Teaching Science and Health from a feminist perspective. (Pergamon Press: Nueva York).

SMAIL, B., 1991. Cómolnteresar a las Chicas por las Ciencias. (MEC: Madrid).

SONEIRA VEIGA, G., 1992. ¿Tienen marca de género las Matemáticas?, en Moreno (ed.), Del Silencio a la Palabra, en Coeducación y Reforma Educativa. (Instituto de la Mujer: Madrid), pp. 306-331.

SPEAR, M., 1987. A teacher's view about the importance of Science for boys and girls, en Kelly (ed.), Science for Girls? (Open University Press).

SUBIRATS, M. y BRULLET, C., 1988. Rosa y Azul. La transmisión de los géneros en la Escuela mixta. (Instituto de la Mujer: Madrid).

WHYTE, J., 1986. Girls into Science and Technology. (Routledge and Kean Paul: London). 


\section{ANEXO 1}

\section{TEXTOS DE CIENCIAS EXPERIMENTALES; PLANTILLA DE ANÁLISIS}

\section{DATOS DE IDENTIFICACIÓN}

Título

Materia

Curso

Editorial

Año

Autoras/Autores

Mujeres

Varones.

\section{CONTENIDOS}

1.1. Citas de científicas y científicos

Mujeres

Varones

en texto en anexos en texto ent anexos

\subsection{Tratamiento Marie Curie}

(M. Curie y su esposo $\square$ M. y P. Curie \P. Curie y su esposa

1.3. Tratamiento de ambos sexos

(] equilibrada $\square$ varón como norma . 7 otras ejemplos y páginas

1.4. Relevancia de contenidos: en conexión con intereses

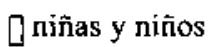

sobre todo niños

ejemplos y páginas

\section{LENGUAJE}

2.1. Coeducado Identificación de varón con genérico

$\begin{array}{lll}\text { ser humano } & \text { tl hombre } & \text { cientificos } \\ \text { individuos } & \text { alumno } & \text { profesor } \\ \text { persona } & \text { niño } & \text { geólogos } \\ \text { organismo humano } & \text { hijo } & \text { investigadores }\end{array}$

\subsection{Estereotipos sexistas}

ejemplos y páginas

\section{ILUSTRACIONES}

\subsection{Proporción mujeres/varones}

$\square$ total ilustr. seres humanos. C]juntos los dos sexos.

Dujeres.

Q varones.

\subsection{Actividades conectadas a experiencias}

¿ niñas y niños

1] sobre todo niños

ejemplos y páginas

\section{CUERPO HUMANO}

\subsection{Siluetas}

$\square$ neutras o dos sexos

$\square$ sil. femeninas...... ] sil. masculinas.......

4.2. Orden del aparato reproductor:
$\square$ alternan
$\square$ masc. fem
$\square$ fem. masc
$\square$ Orden de gametogénesis: $\square$ masc. fem $\square$ fem. masc

\subsection{Contenidos en el tema de la reproducción}

* Sexualidad vinculada sólo a reproducción *otras

* Medios anticonceptivos sí.... no.....

* Estereotipos y prejuicios (ban̈o/regla, masturbación): ]refuerza 0 nomenciona [] critica

* Mención sólo menopausia *tamb. andropausia

* Hormonas sexuales: 1 fern. y masc. Dsolomasculinas

* Presentación clítoris/pene $\square$ independiente $\square$ homólogo de

* Enfermedades de transmisión sexual: sí.... no.....

* Nuevas tecnologías de reproducción sí.... no.....

4.4. Bases biológicas diferencias en roles

Orefuerza Domenciona Deritica

\section{PROBLEMAS}

5.1. [Enunciados coeducados...... [ ] sólo varones.......

5.2. $\square$ Intereses de ambos sexos.......

[] Intereses masculinos.......

6. PRÁCTICAS

6.1. Intereses de ambos sexos.......

DIntereses masculinos.......

\section{OTROS ASPECTOS}

